\title{
Measuring affective temperaments: a systematic review of validation studies of the Temperament Evaluation in Memphis Pisa and San Diego (TEMPS) instruments
}

\author{
Liana R. Elias ${ }^{\mathrm{a}}$, Cristiano A. Köhler ${ }^{\mathrm{a}}$, Brendon Stubbs ${ }^{\mathrm{b}, \mathrm{c}, \mathrm{d}}$, Beatriz R. Maciel ${ }^{\mathrm{a}}$, Lígia \\ M. Cavalcante ${ }^{\mathrm{a}}$, Antonio M.O. Vale ${ }^{\mathrm{a}}$, Xénia Gonda ${ }^{\mathrm{e}, \mathrm{f,g}}$, João Quevedo ${ }^{\mathrm{h}, \mathrm{i}, \mathrm{j}, \mathrm{k}}$, Thomas N. Hyphantis , $^{\mathrm{l}}$ \\ Jair C. Soares ${ }^{\mathrm{j}, \mathrm{m}}$, Eduard Vieta ${ }^{\mathrm{n}}$, André F. Carvalho ${ }^{\mathrm{a}, \mathrm{o}, *}$
}

a Translational Psychiatry Research Group and Department of Clinical Medicine, Federal University of Ceará, Fortaleza, CE, Brazil

b Physiotherapy Department, South London and Maudsley NHS Foundation Trust, Denmark Hill, London SE5 8AZ, United Kingdom

${ }^{\mathrm{c}}$ Health Service and Population Research Department, Institute of Psychiatry, Psychology and Neuroscience, King's College London, De Crespigny Park, London Box SE5 8AF, United Kingdom

${ }^{\mathrm{a}}$ Faculty of Health, Social care and Education, Anglia Ruskin University, Bishop Hall Lane, Chelmsford CM1 1SQ, United Kingdom

e Department of Pharmacodynamics, Faculty of Pharmacy, Semmelweis University, Budapest, Hungary

${ }^{\mathrm{f}}$ MTA-SE Neuropsychopharmacology and Neurochemistry Research Group, Hungarian Academy of Sciences, Semmelweis University, Budapest, Hungary

${ }^{\mathrm{g}}$ Department of Psychiatry and Psychotherapy, Kutvolgyi Clinical Center, Semmelweis University, Budapest, Hungary

h Laboratory of Neurosciences, Graduate Program in Health Sciences, Health Sciences Unit, University of Southern Santa Catarina, Criciuma, SC 88806-000, Brazil

${ }^{\mathrm{i}}$ Center for Translational Psychiatry, Department of Psychiatry and Behavioral Sciences, Medical School, The University of Texas Health Science Center at Houston, Houston, TX, USA

${ }^{\mathrm{j}}$ Center of Excellence on Mood Disorders, Department of Psychiatry and Behavioral Sciences, Medical School, The University of Texas Health Science Center at Houston, Houston, TX, USA

${ }^{\mathrm{k}}$ Neuroscience Graduate Program, Graduate School of Biomedical Sciences, The University of Texas Health Science Center at Houston, Houston, TX, USA

${ }^{1}$ Department of Psychiatry, Faculty of Medicine, School of Health Sciences, University of Ioannina, Ioannina 45110, Greece

${ }^{m}$ Department of Psychiatry and Behavioral Sciences, The University of Texas Health Science Center at Houston, Houston, TX, USA

n Bipolar Disorders Program, Department of Psychiatry and Psychology, Institute of Neuroscience, Hospital Clinic, University of Barcelona, IDIBAPS, CIBERSAM, Barcelona, Catalonia, Spain

'Institute for Clinical Research and Education in Medicine, I.R.E.M., Padova, Italy

\section{A R T I C L E I N F O}

\section{Keywords:}

Affective temperaments

Mood disorders

Validation

Temperament in Memphis Pisa and San Diego

Affective disorders

Psychiatry

\begin{abstract}
A B S T R A C T
Background: The assessment of affective temperaments has provided useful insights for the psychopathological understanding of affective disorders and for the conceptualization of bipolar spectrum disorders. The Temperament in Memphis Pisa and San Diego (TEMPS) instrument has been widely used in research, yet its psychometric properties and optimal factor structure are unclear.

Methods: The PubMed/MEDLINE, PsycINFO, and EMBASE electronic databases were searched from inception until March 15th, 2016. Validation peer-reviewed studies of different versions of the TEMPS performed in adult samples were considered for inclusion.

Results: Twenty-seven studies $(N=20,787)$ met inclusion criteria. Several versions of the TEMPS have been validated in 14 languages across 15 countries. The 110-item self-reported version of the TEMPS has been the most studied version. Most studies (50\%) supported a five factor solution although few studies performed confirmatory factor analyses. A five-factor solution has consistently been reported for the 39-item version of the TEMPS-A. Overall, evidence indicates that different versions of the TEMPS have adequate internal consistency reliability, while the TEMPS-A-110 version has acceptable test-retest reliability. The methodological quality of included studies varied.

Limitations: A meta-analysis could not be performed due to the heterogeneity of settings and versions of the TEMPS utilized.
\end{abstract}

\footnotetext{
* Correspondence to: Department of Clinical Medicine, Faculty of Medicine, Federal University of Ceará, Rua Prof. Costa Mendes, 1608, $4^{\circ}$ andar, 60430-040, Fortaleza, CE, Brazil.

E-mail addresses: andrefc7@terra.com.br, andrefc7@hotmail.com (A.F. Carvalho).
} 
Conclusions: Different versions of the TEMPS have been validated across different cultures. The short 39-item version of the TEMPS-A holds promise and merits further investigation. Culture-bound factors may influence the expression and/or assessment of affective temperaments with the TEMPS.

\section{Introduction}

Notwithstanding early versions of the theory of humors might have existed in early Egypt or Mesopotamia the theory was established by the School of Cos and specifically by Polybos, a pupil of Hippocrates

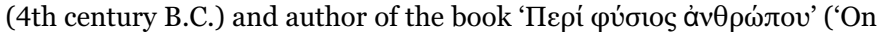
the Nature of Man'). This theory is a precursor of the temperament theory, which originally described four temperament types (in Greek 'crasis') according to the predominance of a particular humor: the Choleric (yellow bile from the liver; cyclothymic), the Sanguine (blood from the heart; hyperthymic) the Melancholic (black bile from the kidneys; depressive) and the Phlegmatic (phlegm from the lungs; selfcontent) (Fountoulakis et al., 2016).

Approaching a century ago, the seminal work of Kraepelin initially recognized four basic affective dispositions namely depressive, manic, cyclothymic, and irritable, which he believed could represent subclinical forms, and in many circumstances precursors of affective psychoses (Kraepelin, 1921). He proposed that these affective temperaments had their roots in adolescence (Kraepelin, 1921). A renewed interest in the field of affective temperaments emerged with the operationalization of this construct by Akiskal and coworkers (Akiskal and Akiskal, 2005; Akiskal and Mallya, 1987; Matsumoto et al., 2005), which was primarily based on the previous work from Kraepelin (Kraepelin, 1921) and Kretschmer (Kretschmer, 1936), and on clinical and theoretical observations.

Affective temperaments are thought to be a 'heritable' and relatively stable phenotype over lifespan (Kawamura et al., 2010). In order to capture affective temperaments, the Temperament Evaluation in Memphis Pisa and San Diego (TEMPS) was initially developed as the TEMPS-I administered in an interview format (Akiskal et al., 1998; Placidi et al., 1998), and as a self-report questionnaire TEMPS-A with 110 (for men) or 109 (for women) items (Akiskal et al., 2005b). The TEMPS-A yields a five-factor structure, which corresponds to the five predominant affective temperaments namely depressive, anxious, irritable, hyperthymic, and cyclothymic (Akiskal et al., 2005b). More recently, more succinct versions of the TEMPS-A have been developed to improve compliance of respondents while capturing its five-factor underlying constructs (Erfurth et al., 2005; Preti et al., 2010; Woodruff et al., 2011). Different versions of the TEMPS instrument have been translated and/or validated in more than twenty-five languages, including but not limited to Italian (Pompili et al., 2008), French (Krebs et al., 2006), German (Erfurth et al., 2005), Brazilian Portuguese (Woodruff et al., 2011), Japanese (Matsumoto et al., 2005), Turkish (Vahip et al., 2005), Polish (Borkowska et al., 2010), Hungarian (Rozsa et al., 2008), Lebanese Arabic (Karam et al., 2005), Spanish-Barcelona (Sanchez-Moreno et al., 2005), Spanish-Buenos Aires (Vazquez et al., 2007), and Lisbon-Portuguese versions (Figueira et al., 2008). Furthermore, the TEMPS has been the most widely used set of instruments to measure affective temperaments.

The concept of predominant affective temperament is instrumental for the definition and characterization of the so-called bipolar spectrum (Akiskal and Pinto, 1999; Angst, 2007; Ghaemi and Dalley, 2014). In addition, certain predominant affective temperaments may confer a higher risk for the emergence of suicidality in patients with mood disorders (Baldessarini et al., 2016; Perugi et al., 2012), and also may contribute to the emergence of both depressive (Carvalho et al., 2013) and somatic (Hyphantis et al., 2013) symptoms. Beyond their role as precursors, risk factors, and/or moderators/mediators of distinct manifestations of mental disorders, affective temperaments may also influence creativity (Akiskal and Akiskal, 2007; Srivastava et al., 2010) and professional choice (Akiskal et al., 2005d; Schmidt et al., 2010).

Despite the widespread use of the TEMPS in numerous settings across different cultural milieus, to the best of our knowledge no previous systematic review has synthesized the psychometric properties of the several versions of this instrument across the various validation studies. Thus, we conducted a systematic review of validation studies of the TEMPS instrument.

\section{Methods}

\subsection{Search strategy}

This systematic review followed an a priori defined yet unpublished protocol. The electronic databases PubMed/MEDLINE, PsycInfo, and EMBASE were searched from inception up until March 15th, 2016. The detailed search string used in this systematic review is provided in the supplementary online material that accompanies the online version of this article. In addition, this search strategy was augmented through tracking the citations of eligible articles in Google Scholar (Bakkalbasi et al., 2006). We followed the Preferred Reporting Items for Systematic Reviews and Meta-Analyses (PRISMA) statement (Moher et al., 2009).

\subsection{Eligibility criteria}

Validation studies of different versions of the TEMPS conducted in adult samples (i.e. aged $\geq 21$ years old) from any setting (e.g. university students, general population, psychiatric outpatients, etc.) were considered for inclusion. Only original peer-reviewed articles were included. No language restriction was applied. Studies whose samples included children/adolescents, as well as case reports and review articles were excluded. Studies that did not report psychometric parameters of different versions of the TEMPS were also excluded. We included studies from both clinical and non-clinical samples. No restriction was applied regarding psychiatric diagnoses.

\subsection{Study selection}

The titles/abstracts of unique references were independently screened for eligibility by two investigators (LRE, LMC or BRM). Afterwards, the full-texts of selected reports were obtained, and the same authors independently reviewed each article to determine its eligibility. Whenever a consensus could not be reached, a third author (CAK or AFC) made the final decision regarding inclusion.

\subsection{Data extraction}

Data from included references were independently extracted using a standardized form by two authors (LRE, BRM or LMC). Discrepancies were resolved through consensus. The following information was extracted for each included study: author, year, version of the instrument (and any necessary modifications from the original version of the instrument), translation strategy, study design, sample size, gender distribution (\% females), age of the samples (mean \pm standard deviation), setting, and sample characteristics, version of the TEMPS, and a description of modifications performed in the original version of the instrument. When studies included overlapping samples, we considered only the one with the largest sample size. However, we included separate reports involving the same sample whenever different psychometric properties of the TEMPS were provided. Authors were contacted to provide additional data (i.e., in case relevant data 


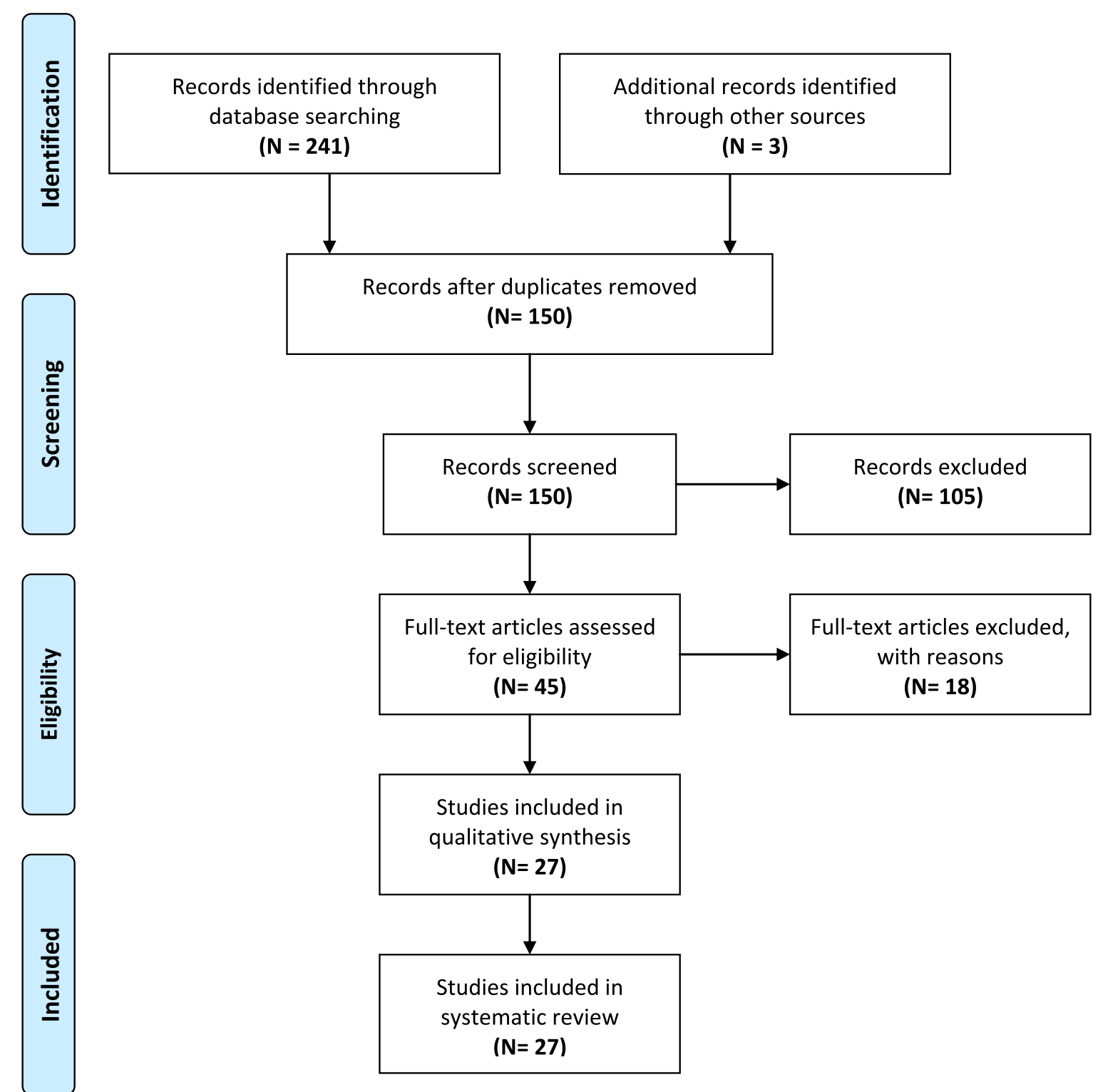

Fig. 1. PRISMA flowchart of study selection.

were not presented in the original report). The following psychometric properties were extracted whenever available: reliability (test-retest reliability and internal consistency reliability), concurrent validity, and construct validity (e.g., exploratory factor analysis and confirmatory factor analysis).

The methodological quality of included studies were assessed through an a priori devised tool (see Supplementary material). Scores could vary from 0 to 8 ; higher scores indicate better methodological quality.

\section{Results}

The initial search yielded 241 hits, while 3 additional hits were derived from the citation tracking of included articles in Google Scholar. After exclusion of duplicates, 150 unique references underwent title/abstract screening of which 46 were selected for full-text review. Of those 46 references, 18 were excluded with reasons (see Table S1 in Supplementary material that accompanies the online version of this article). Finally, 27 articles (Akiskal et al., 2005a, 2005b, 2005c; Akiyama et al., 2005; Bloink et al., 2005; Borkowska et al., 2010; Dolenc and Sprah, 2011; Dolenc et al., 2013; Erfurth et al., 2005; Fountoulakis et al., 2014; Hinic et al., 2013; Karam et al., 2005;
Kawamura et al., 2010; Lin et al., 2013; Maremmani et al., 2011; Matsumoto et al., 2005; Naderer et al., 2015; Pompili et al., 2008; Preti et al., 2015, 2013, 2010; Ristic-Ignjatovic et al., 2014; Rozsa et al., 2006; Vahip et al., 2005; Victor et al., 2006; Woodruff et al., 2011; Yuan et al., 2015) were included in this systematic review. The PRISMA flowchart of study selection for this systematic review is provided in Fig. 1.

\subsection{Characteristics and methodological quality of included studies}

Overall, this systematic review included 27 studies comprising data from 20,787 participants. All studies followed a cross-sectional design. The sample size of included studies varied from 62 to 1450 participants (mean=769.9; $\mathrm{SD}=379.5$ ), while the age of participants across studies was $31.4 \pm 13.5$ (mean \pm SD), and the gender distribution (\% of females) varied from $7.5 \%$ to $75.6 \%$ (mean=54.9; $\mathrm{SD}=14.3$ ). Regarding settings, eight studies (29.6\%) enrolled samples derived from mixed settings (e.g., undergraduate students, general population, etc.), while the remaining studies enrolled participants from a single setting (10, 37.0\% studies enrolled university students; $3,11.1 \%$ studies enrolled clinical samples; 6, 22.2\% studies enrolled general 


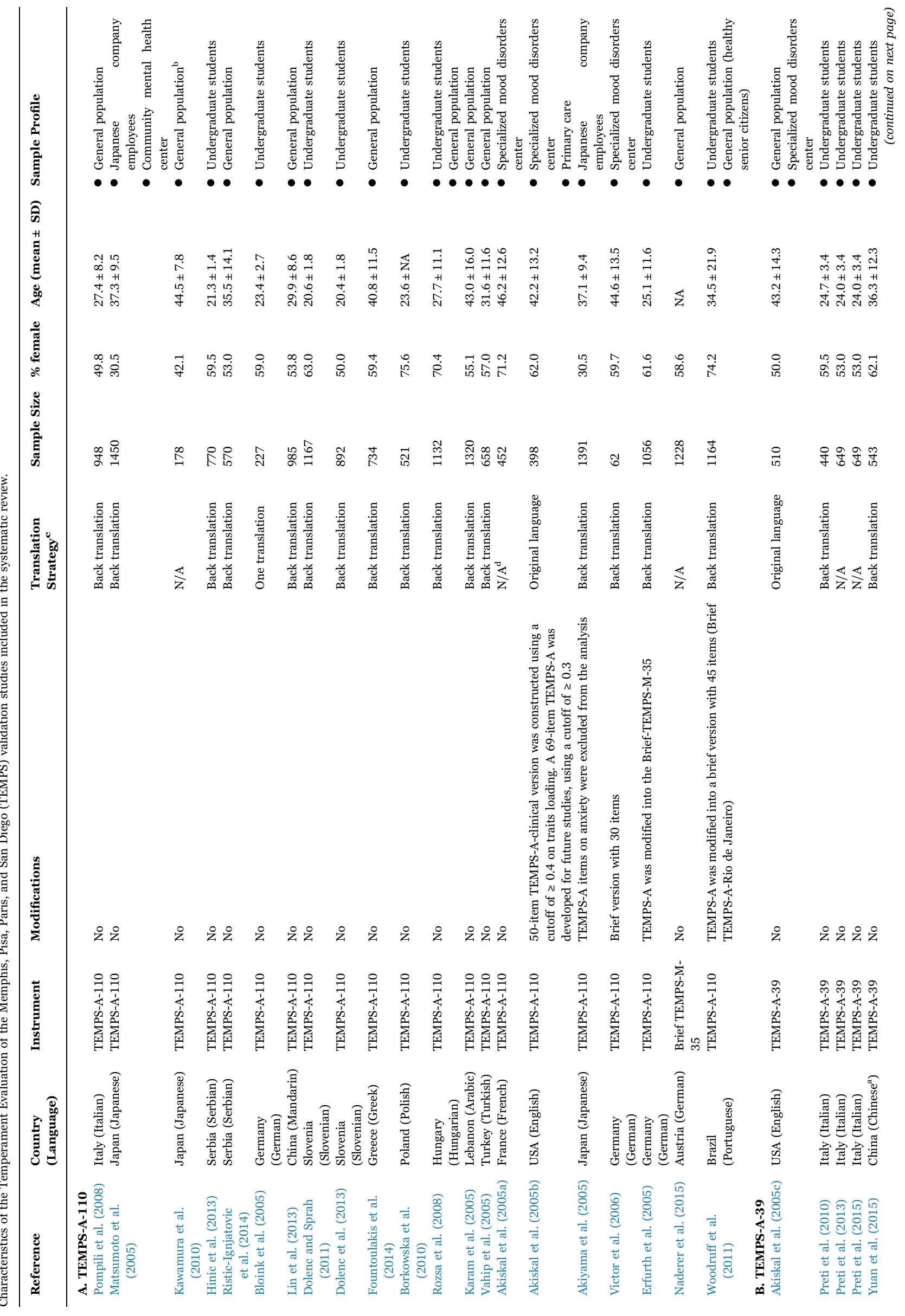


population samples). This systematic review included validation studies of different TEMPS versions (vide infra) in 14 different languages (English, Italian, Japanese, Serbian, German, Chinese, Slovenian, Greek, Polish, Portuguese, Hungarian, French, Arabic, Turkish) from 15 countries (USA, Italy, Japan, Serbia, Germany, Austria, China, Slovenia, Greece, Poland, Brazil, Hungary, France, Lebanon, Turkey). The characteristics of included studies are provided in Table 1.

Methodological quality scores of included studies varied from 2 to 8 (median=5). The breakdown of each score of the methodological assessment tool is given in Supplementary Table S2.

\subsection{Different versions of the TEMPS instrument}

Fifteen studies used the original TEMPS-A-110 version (Akiskal et al., 2005b; Bloink et al., 2005; Borkowska et al., 2010; Dolenc and Sprah, 2011; Dolenc et al., 2013; Fountoulakis et al., 2014; Hinic et al., 2013; Karam et al., 2005; Kawamura et al., 2010; Lin et al., 2013; Matsumoto et al., 2005; Pompili et al., 2008; Ristic-Ignjatovic et al., 2014; Rozsa et al., 2008; Vahip et al., 2005) (Table 1A). This version was modified in five studies. Akiskal et al. (2005b) validated a shorter version for use in clinical samples, using factor analysis to extract a 4factor scale and obtaining 50-item and 69-item versions. Akiyama et al. (2005) developed a 39-item version that excluded the anxiety factor because the anxiety temperament was not included in the original description of "fundamental states" by Kraepelin (1921). Victor et al. (2006) developed a brief German version with 30 items; this version was developed after factor analysis. Erfurth et al. (2005) developed another brief German version with 35 items, denominated the Münster translation (Brief TEMPS-M-35). These authors changed the original scoring scale to a Likert-type 1-5 scale, and used factor analysis to select the items that loaded into the five predominant affective temperaments (i.e., hyperthymic, cyclothymic, irritable, anxious and euthymic). This version (i.e., TEMPS-M-35) was further validated by Naderer et al. (2015) in Austria. Finally, Woodruff et al. (2011) translated the original 110-version of the TEMPS and used PCA to develop a 45-item Brazilian Portuguese of the TEMPS (brief TEMPS-ARio de Janeiro).

Five studies used the short TEMPS-A-39 version (Akiskal et al., 2005c; Preti et al., 2015, 2013, 2010; Yuan et al., 2015). See Table 1 B. This version was developed as an alternative to the full TEMPS-A-110 aiming to improve the compliance of respondents (Akiskal et al., 2005c).

A single study was based on the TEMPS-I version (Maremmani et al., 2011) (Table 1C). This version is a 61-item TEMPS-A version (developed in Pisa) directly derived from the Italian version of the TEMPS-I, and is referred to as the TEMPS-A[P].

\subsection{Factor structure}

Principal component analysis (PCA) was performed in 16 studies that validated the TEMPS-A-110 (Table 2). All studies used the Varimax rotation method, with the exception of the study by Dolenc et al. (2013), which used the Oblimin rotation method. Most studies $(k=8 ; 50 \%)$ confirmed the 5-factor structure of the TEMPS-A-110. Ristic-Ignjatovic et al. (2014) found six factors; these authors obtained an anxious-cognitive and an anxious-somatic factor.

Three studies found only two factors. In the study by Lin et al. (2013) the depressive, cyclothymic, irritable and anxious temperaments loaded on a first factor (57.8\% of the variance was explained by this factor), while the second factor loaded onto the hyperthymic temperament (which explained $19.3 \%$ of the variance). The study by Rozsa et al. (2006) also derived two factors, which explained $47.9 \%$ and $23.7 \%$ of the variance respectively. Finally, Borkowska et al. (2010) observed that the cyclothymic, irritable and anxious temperaments loaded predominantly on the first factor (53.6\% of the variance), while depressive hyperthymic temperaments loaded on the second factor 
Table 2

Factor structure and internal consistency obtained in TEMPS validation studies.

\begin{tabular}{|c|c|c|c|c|c|c|c|}
\hline \multirow[t]{2}{*}{ Reference } & \multicolumn{5}{|c|}{ Factor structure } & \multirow{2}{*}{\multicolumn{2}{|c|}{$\begin{array}{l}\text { Internal consistency (per } \\
\text { factor) }\end{array}$}} \\
\hline & Method & $\begin{array}{l}\text { Rotation } \\
\text { method }\end{array}$ & $\begin{array}{l}\text { Number of } \\
\text { factors }\end{array}$ & $\begin{array}{l}\text { Loading factor } \\
\text { cutoff }\end{array}$ & $\begin{array}{l}\text { Variance of the } \\
\text { solution (\%) }\end{array}$ & & \\
\hline \multicolumn{8}{|l|}{ A. TEMPS-A-110 } \\
\hline Naderer et al. (2015) ${ }^{\mathbf{e}}$ & PCA & Varimax & 5 & 0.50 & 50.8 & $\begin{array}{l}\text { - Depressive: } 0.87 \\
\text { - Cyclothymic: } 0.86 \\
\text { - Hyperthymic: } 0.82 \\
\text { - Irritable: } 0.80 \\
\text { - Anxious: } 0.75\end{array}$ & \\
\hline $\begin{array}{l}\text { Ristic-Ignjatovic et al. } \\
\text { (2014) }\end{array}$ & PCA & Varimax & 6 & 0.50 & 44.4 & $\begin{array}{l}\text { - Depressive: } 0.73 \\
\text { - } \text { Cyclothymic: } 0.80 \\
\text { - Hyperthymic: } 0.74 \\
\text { - Irritable: } 0.74 \\
\text { - Anxious } \mathrm{Cog}^{\mathrm{a}}: 0.80 \\
\text { - Anxious } \mathrm{Som}^{\mathrm{a}}: 0.73\end{array}$ & \\
\hline Fountoulakis et al. (2014) & NP & NP & $\mathrm{NP}$ & NP & NP & $\begin{array}{l}\text { - Depressive: } 0.72 \\
\text { - Cyclothymic: } 0.83 \\
\text { - Hyperthymic: } 0.80 \\
\text { - Irritable: } 0.80 \\
\text { - Anxious: } 0.88\end{array}$ & \\
\hline Hinic et al. (2013) & PCA & Varimax & 5 & 0.40 & 31.7 & $\begin{array}{l}\text { - Depressive: } 0.76 \\
\text { - Cyclothymic: } 0.69 \\
\text { - Hyperthymic: } 0.73 \\
\text { - Irritable: } 0.70 \\
\text { - Anxious: } 0.73\end{array}$ & \\
\hline Lin et al. (2013) & PCA & Varimax & 2 & 0.30 & $\begin{array}{l}\text { Factor I: } 57.8^{\mathrm{b}} \\
\text { Factor II: } 19.3^{\mathrm{b}}\end{array}$ & $\begin{array}{l}\text { - Depressive: } 0.68 \\
\text { - Cyclothymic: } 0.85 \\
\text { - Hyperthymic: } 0.82 \\
\text { - Irritable: } 0.83 \\
\text { - Anxious: } 0.87\end{array}$ & \\
\hline Dolenc et al. (2013) & PCA & Varimax Oblimin & 5 & 0.30 & 24.9 & $\begin{array}{l}\text { Males: } \\
\text { - Depressive: } 0.55 \\
\text { - Cyclothymic: } 0.72 \\
\text { - Hyperthymic: } 0.76 \\
\text { - Irritable: } 0.77 \\
\text { - Anxious: } 0.80 \\
\text { Females: } \\
\text { - Depressive: } 0.69 \\
\text { - Cyclothymic: } 0.77 \\
\text { - Hyperthymic: } 0.75 \\
\text { - Irritable: } 0.80 \\
\text { - Anxious: } 0.84\end{array}$ & \\
\hline Dolenc and Sprah (2011) & PCA & N/A & 5 & $\mathrm{~N} / \mathrm{A}$ & $\mathrm{N} / \mathrm{A}$ & $\begin{array}{l}\text { - Depressive: } 0.66 \\
\text { - Cyclothymic: } 0.76 \\
\text { - Hyperthymic: } 0.76\end{array}$ & \\
\hline & & & & & & $\begin{array}{l}\text { Irritable: } 0.77 \text { (males), } \\
\text { (females) } \\
\text { - Anxious: } 0.84\end{array}$ & 0.79 \\
\hline Woodruff et al. $(2011)^{\mathbf{e}}$ & PCA & Varimax & 6 & 0.35 & 26.5 & $\begin{array}{l}\text { - Anxious: } 0.67 \\
\text { - Worrying: } 0.81 \\
\text { - Cyclothymic: } 0.74 \\
\text { - Irritable: } 0.74 \\
\text { - Depressive: } 0.72 \\
\text { - Hyperthymic: } 0.70\end{array}$ & \\
\hline Borkowska et al. (2010) & PCA & Varimax & 2 & 0.60 & 75.1 & $\begin{array}{l}\text { - Depressive: } 0.70 \\
\text { - Cyclothymic: } 0.77 \\
\text { - Hyperthymic: } 0.75 \\
\text { - Irritable: } 0.76 \\
\text { - Anxious: } 0.83\end{array}$ & \\
\hline Pompili et al. (2008) & PCA & Varimax & 3 & 0.10 & 19.7 & $\begin{array}{l}\text { - Dysthymic-Cyclothymic- } \\
\text { Anxious: } 0.89 \\
\text { - Irritable: } 0.77 \\
\text { - Hyperthymic: } 0.74\end{array}$ & \\
\hline Rozsa et al. (2006) & PCA & Varimax & 2 & 0.40 & $\begin{array}{l}\text { Factor I: } 47.9^{\mathrm{c}} \\
\text { Factor II: } 23.6^{\mathrm{c}}\end{array}$ & $\begin{array}{l}\text { - Depressive: } 0.65 \\
\text { - Cyclothymic: } 0.8 \\
\text { - Hyperthymic: } 0.79 \\
\text { - Irritable: } 0.75 \\
\text { - Anxious: } 0.81\end{array}$ & \\
\hline Akiyama et al. (2005) ${ }^{\mathbf{e}}$ & PCA & Varimax & 3 & 0.42 & 19.1 & $\mathrm{~N} / \mathrm{A}$ & \\
\hline Erfurth et al. (2005) & PCA & Varimax & 5 & 0.50 & $\mathrm{~N} / \mathrm{A}$ & $\begin{array}{l}\text { - Depressive: } 0.85 \\
\text { - } \text { Cyclothymic: } 0.80 \\
\text { - Hyperthymic: } 0.77 \\
\text { - } \text { Irritable: } 0.74 \\
\text { - Anxious: } 0.71\end{array}$ & 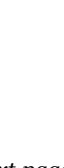 \\
\hline
\end{tabular}


Table 2 (continued)

\begin{tabular}{|c|c|c|c|c|c|c|}
\hline \multirow[t]{2}{*}{ Reference } & \multicolumn{5}{|c|}{ Factor structure } & \multirow{2}{*}{$\begin{array}{l}\text { Internal consistency (per } \\
\text { factor) }\end{array}$} \\
\hline & Method & $\begin{array}{l}\text { Rotation } \\
\text { method }\end{array}$ & $\begin{array}{l}\text { Number of } \\
\text { factors }\end{array}$ & $\begin{array}{l}\text { Loading factor } \\
\text { cutoff }\end{array}$ & $\begin{array}{l}\text { Variance of the } \\
\text { solution (\%) }\end{array}$ & \\
\hline Akiskal et al. (2005a) & PCA & $\mathrm{N} / \mathrm{A}$ & $3^{\mathrm{d}}$ & $\mathrm{N} / \mathrm{A}$ & $\mathrm{N} / \mathrm{A}$ & $\mathrm{N} / \mathrm{A}$ \\
\hline Akiskal et al. (2005b) & PCA & Varimax & 4 & 0.30 & 28.5 & $\begin{array}{l}\text { - Cyclothymic: } 0.88 \\
\text { - Irritable: } 0.84 \\
\text { - Hyperthymic: } 0.81 \\
\text { - Dysthymic: } 0.76\end{array}$ \\
\hline Karam et al. (2005) & PCA & Varimax & 5 & 0.30 & N/A & $\begin{array}{l}\text { - Depressive: } 0.66 \\
\text { - Cyclothymic: } 0.83 \\
\text { - Hyperthymic: } 0.82 \\
\text { - Irritable: } 0.76 \\
\text { - Anxious: } 0.88\end{array}$ \\
\hline Vahip et al. (2005) & PCA & Varimax & 5 & 0.20 & N/A & $\begin{array}{l}\text { - Depressive: } 0.77 \\
\text { - Cyclothymic: } 0.85 \\
\text { - Hyperthymic: } 0.80 \\
\text { - Irritable: } 0.82 \\
\text { - Anxious: } 0.84\end{array}$ \\
\hline Matsumoto et al. (2005) & NP & NP & NP & NP & $\mathrm{NP}$ & $\begin{array}{l}\text { - Depressive: } 0.69 \\
\text { - Cyclothymic: } 0.84 \\
\text { - Hyperthymic: } 0.79 \\
\text { - Irritable: } 0.83 \\
\text { - Anxious: } 0.87\end{array}$ \\
\hline Bloink et al. (2005) & NP & NP & NP & NP & $\mathrm{NP}$ & $\begin{array}{l}\text { - Depressive: } 0.62 \\
\text { - Cyclothymic: } 0.73 \\
\text { - Hyperthymic: } 0.71 \\
\text { - Irritable: } 0.71 \\
\text { - Anxious: } 0.76\end{array}$ \\
\hline Victor et al. (2006) & NP & NP & NP & NP & NP & $\begin{array}{l}\text { - Depressive: } 0.8 \\
\text { - Cyclothymic: } 0.81 \\
\text { - Hyperthymic: } 0.83 \\
\text { - Irritable: } 0.75 \\
\text { - Anxious: } 0.84\end{array}$ \\
\hline Kawamura et al. (2010) & NP & NP & NP & NP & NP & NP \\
\hline \multicolumn{7}{|l|}{ B. TEMPS-A-39 } \\
\hline Akiskal et al. (2005c) & PCA & Varimax & 5 & 0.35 & 46.0 & $\begin{array}{l}\text { - Cyclothymic: } 0.91 \\
\text { - Depressive: } 0.81 \\
\text { - Irritable: } 0.77 \\
\text { - Hyperthymic: } 0.76 \\
\text { - Anxious: } 0.67\end{array}$ \\
\hline Preti et al. (2010) & PCA & Promax & 5 & 0.30 & 58.6 & $\begin{array}{l}\text { - Depressive: } 0.72 \\
\text { - Cyclothymic: } 0.79 \\
\text { - Hyperthymic: } 0.75 \\
\text { - Irritable: } 0.72 \\
\text { - Anxious: } 0.71\end{array}$ \\
\hline Preti et al. (2013) & NP & NP & NP & NP & NP & $\begin{array}{l}\text { - Depressive: } 0.72 \\
\text { - Cyclothymic: } 0.83 \\
\text { - Hyperthymic: } 0.70 \\
\text { - Irritable: } 0.65 \\
\text { - Anxious: } 0.67\end{array}$ \\
\hline Preti et al. (2015) & NP & NP & NP & NP & NP & $\begin{array}{l}\text { - Depressive: } 0.73 \\
\text { - Cyclothymic: } 0.82 \\
\text { - Hyperthymic: } 0.70 \\
\text { - Irritable: } 0.65 \\
\text { - Anxious: } 0.67\end{array}$ \\
\hline Yuan et al. (2015) & PCA & Promax & 5 & 0.30 & 43.4 & $\begin{array}{l}\text { - Depressive: } 0.80 \\
\text { - Cyclothymic: } 0.89 \\
\text { - Hyperthymic: } 0.72 \\
\text { - Irritable: } 0.71 \\
\text { - Anxious: } 0.70\end{array}$ \\
\hline \multicolumn{7}{|l|}{ C. TEMPS-I } \\
\hline Maremmani et al. (2011) & NP & NP & NP & NP & $\mathrm{NP}$ & NP \\
\hline
\end{tabular}

Abbreviations: $\mathbf{N} / \mathbf{A}=$ data not available; $\mathbf{N P}=$ not performed; $\mathbf{P C A}=$ principal component analysis.

a The Anxious factor was subdivided into Anxious Cognitive and Anxious Somatic.

${ }^{\mathrm{b}}$ Factor I explained $57.8 \%$ of the variance, loading on depressive, cyclothymic, irritable and anxious temperaments; An independent Factor II explained $19.3 \%$ variance, loading on the hyperthymic temperament.

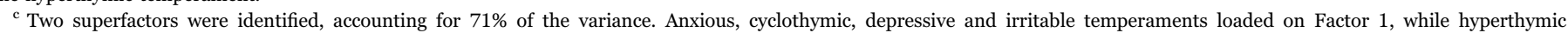
temperament loaded on Factor II.

$\mathrm{d}$ Before rotation, each subscale loaded into one global factor in which nearly all items are well represented (loadings $>0.3$.).

e Refers to a modification of the original TEMPS version. See Table 1 for further details. 
Table 3

Concurrent validity obtained in TEMPS validation studies.

\begin{tabular}{|c|c|c|c|c|c|c|}
\hline \multirow[t]{2}{*}{ Reference } & \multirow{2}{*}{$\begin{array}{l}\text { Scales } \\
\text { used }\end{array}$} & \multicolumn{5}{|c|}{ Correlation with TEMPS temperament score } \\
\hline & & Depressive & Cyclothymic & Hyperthymic & Irritable & Anxious \\
\hline $\begin{array}{r}\text { Preti et al. } \\
\text { (2015) }\end{array}$ & SPQ & $\begin{array}{l}\text { IF: } 0.35 \\
\text { - } \mathrm{ESA}: 0.40 \\
\text { - } \mathrm{MT}: 0.15 \\
\text { - UPE: } 0.20 \\
\text { OB: } 0.27 \\
\text { - NCF: } 0.33 \\
\text { OS: } 0.40 \\
\text { - } \mathrm{CA}: 0.39 \mathrm{~S}: 0.53\end{array}$ & $\begin{array}{l}\text { - IF: } 0.47 \\
\text { ESA: } 0.41 \\
\text { - MT: } 0.35 \\
\text { UPE: } 0.41 \\
\text { OB: } 0.36 \\
\text { - NCF: } 0.29 \\
\text { OS: } 0.49 \\
\text { - CA: } 0.35 \text { S: } 0.51\end{array}$ & $\begin{array}{l}\text { - } \text { IF: } 0.08 \\
\text { - } \text { ESA: }-0.29 \\
\text { - } \text { MT: } 0.07 \\
\text { UPE: } 0.07 \\
\text { OB: } 0.14 \\
\text { NCF: }-0.25 \\
\text { - OS: }-0.02 \\
\text { - } \text { CA: }-0.17 \mathrm{~S}:-0.004\end{array}$ & $\begin{array}{l}\text { IF: } 0.31 \\
\text { ESA: } 0.19 \\
\text { MT: } 0.18 \\
\text { UPE: } 0.23 \\
\text { OB: } 0.26 \\
\text { NCF: } 0.16 \\
\text { OS: } 0.29 \\
\text { CA: } 0.20 \mathrm{~S}: 0.38\end{array}$ & $\begin{array}{l}\text { - IF: } 0.30 \\
\text { ESA: } 0.33 \\
\text { - } \text { MT: } 0.21 \\
\text { - UPE: } 0.24 \\
\text { OB: } 0.19 \\
\text { - NCF: } 0.08 \\
\text { - OS: } 0.31 \\
\text { - CA: } 0.16 \text { S: } 0.36\end{array}$ \\
\hline $\begin{array}{l}\text { Ristic- } \\
\text { Ignjatovic } \\
\text { et al. (2014) }\end{array}$ & TCI-R & $\begin{array}{l}\text { - Novelty seeking: } \\
-0.22 \\
\text { - Harm avoidance: } 0.51 \\
\text { - Reward dependence: } \\
-0.18 \\
\text { - Persistence: }-0.21\end{array}$ & $\begin{array}{l}\text { - Novelty seeking: } 0.25 \\
\text { - Harm avoidance: } 0.40 \\
\text { - Reward dependence: } \\
0.21 \\
\text { - Persistence: }-0.11\end{array}$ & $\begin{array}{l}\text { - Novelty seeking: } 0.41 \\
\text { - Harm avoidance: } \\
-0.47 \\
\text { - } \text { Reward dependence: } \\
0.17 \\
\text { - Persistence: } 0.31\end{array}$ & $\begin{array}{l}\text { - Novelty seeking: } 0.22 \\
\text { - Harm avoidance: } 0.23 \\
\text { - Reward dependence: } \\
-0.18 \\
\text { - Persistence: }-0.19\end{array}$ & $\begin{array}{l}\text { Anxious Cognitive }{ }^{\mathrm{a}} \text { : } \\
\text { Novelty seeking: }-0.21 \mathrm{Harm} \\
\text { avoidance: } 0.48 \text { Reward } \\
\text { dependence: } 0.24 \text { Persistence: } \\
-0.10 \text { Anxious Somatic }{ }^{\mathrm{a}} \text { :Novelty } \\
\text { seeking: }-0.15 \text { Harm avoidance: } \\
0.41 \text { Reward dependence: } \\
0.22 \text { Persistence: }-0.05\end{array}$ \\
\hline \multirow[t]{2}{*}{$\begin{array}{l}\text { Maremmani } \\
\text { et al. (2011) }\end{array}$} & $\begin{array}{l}\text { MMPI } \\
\text { Clinical } \\
\text { Scale }\end{array}$ & $\begin{array}{ll}\text { - } & \text { Hypochondrias: N/A } \\
\text { - } & \text { Depression: N/A } \\
\text { - } & \text { Psychoria: N/A } \\
& \text { Deviation: N/A } \\
\text { - } & \text { Masculinity- } \\
& \text { Femininity: N/A } \\
\text { - Paranoia: N/A } \\
\text { - Psychasthenia: N/A } \\
\text { - Schizophrenia: N/A } \\
\text { - Hypomania: N/A } \\
\text { - Social Introversion: } \\
& 0.12\end{array}$ & $\begin{array}{ll}\text { - } & \text { Hypochondrias: } \\
& -0.16 \\
\text { - } & \text { Depression: N/A } \\
\text { - } & \text { Hysteria: }-0.21 \\
\text { - Psychopathic } \\
\\
\text { - } \text { Deviation: } 0.13 \\
\text { Masculinity- } \\
\text { - Pemininity: N/A } \\
\text { - Paranoia: }-0.20 \\
\text { - Schchasthenia: }-0.26 \\
\text { - Hypophrenia: }-0.25 \\
\text { - Social } \\
\text { Introversion: } 0.29\end{array}$ & $\begin{array}{l}\text { - Hypochondrias: N/A } \\
\text { - } \text { Depression: }-0.18 \\
\text { - Hysteria: N/A } \\
\text { - Psychopathic } \\
\text { Deviation: }-0.01 \\
\text { - Masculinity- } \\
\text { Femininity: N/A } \\
\text { - Paranoia: N/A } \\
\text { - Psychasthenia: N/A } \\
\text { - Schizophrenia: N/A } \\
\text { - Hypomania: } 0.29 \\
\text { - Social Introversion: } \\
-0.37\end{array}$ & $\begin{array}{l}\text { - Hypochondrias: } \\
-0.13 \\
\text { - Depression: }-0.12 \\
\text { - Hysteria: }-0.20 \\
\text { - Psychopathic } \\
\text { Deviation: }-0.02 \\
\text { - Masculinity- } \\
\text { Femininity: N/A } \\
\text { - Paranoia: }-0.15 \\
\text { - Psychasthenia: }-0.24 \\
\text { - Schizophrenia: }-0.19 \\
\text { - Hypomania: } 0.13 \\
\text { - Social Introversion: } \\
\\
0.12\end{array}$ & $\mathrm{~N} / \mathrm{A}$ \\
\hline & $\begin{array}{l}\text { MMPI } \\
\text { Validity } \\
\text { Scale }\end{array}$ & $\begin{array}{ll}- & \text { L: N/A } \\
\text { - } & \mathrm{F}: \mathrm{N} / \mathrm{A} \\
& \mathrm{K}:-0.10\end{array}$ & $\begin{array}{l}-\mathrm{L}:-0.38 \\
\text { - } \mathrm{F}: 0.19 \\
\text { - } \mathrm{K}:-0.48\end{array}$ & $\begin{array}{ll}- & \text { L: } 0.14 \\
- & \text { F: N/A } \\
\text { - } & \text { K: N/A }\end{array}$ & $\begin{array}{l}-\mathrm{L}:-0.20 \\
\text { - F: } 0.15 \\
\text { - } \mathrm{K}:-0.31\end{array}$ & N/A \\
\hline \multirow[t]{2}{*}{$\begin{array}{l}\text { Rozsa et al. } \\
\quad(2006)\end{array}$} & TCI & $\begin{array}{ll}\text { - } & \text { Novelty seeking: } \\
& -0.21^{* *} \\
\text { - } & \text { Harm avoidance: } \\
& 0.47^{*} \\
\text { - } & \text { Reward dependence: } \\
& 0.17 \\
\text { - Persistence: }-0.04 \\
\text { - Self-directedness: - } \\
0.28^{*} \\
\text { - } \text { Cooperativeness: } 0.01 \\
\text { - Self-transcendence: } \\
-0.10\end{array}$ & $\begin{array}{ll}\text { - } & \text { Novelty seeking: } \\
& 0.23^{* * *} \\
\text { - } & \text { Harm avoidance: } \\
& 0.34^{* *} \\
\text { - } & \text { Reward dependence: } \\
& 0.19^{* *} \\
\text { - Persistence: }-0.09 \\
\text { - Self-directedness: } \\
-0.42^{* * *} \\
\text { - } \text { Cooperativeness: } \\
-0.15 \\
\text { - Self-transcendence: } \\
\\
0.22^{* * *}\end{array}$ & $\begin{array}{ll}\text { - } & \text { Novelty seeking: } 0.07 \\
\text { - } & \text { Harm avoidance: } \\
& -0.48^{*} \\
\text { - } & \text { Reward dependence: } \\
& -0.20^{* *} \\
\text { - } & \text { Persistence: } 0.28^{*} \\
\text { - Self-directedness: } \\
\\
0.31^{*} \\
\text { - } \text { Cooperativeness: } 0.0 \\
\text { - Self-transcendence: } \\
0.24^{* *}\end{array}$ & $\begin{array}{l}\text { - Novelty seeking: } 0.18 \\
\text { - Harm avoidance: } 0.16 \\
\text { - Reward dependence: } \\
-0.16 \\
\text { - Persistence: } 0.20^{* *} \\
\text { - Self-directedness: } \\
-0.33^{*} \\
\text { - Cooperativeness: } \\
-0.31^{*} \\
\text { - Self-transcendence: } \\
0.02\end{array}$ & $\begin{array}{l}\text { - Novelty seeking: }-0.03 \\
\text { - Harm avoidance: } 0.57^{*} \\
\text { - Reward dependence: } 0.29^{* * *} \\
\text { - Persistence: } 0.02 \\
\text { - Self-directedness: }-0.43^{*} \\
\text { - Cooperativeness: }-0.04 \\
\text { - Self-transcendence: }-0.11\end{array}$ \\
\hline & $\begin{array}{l}\text { NEO-PI- } \\
\mathrm{R}\end{array}$ & $\begin{array}{l}\text { - Neuroticism: } 0.47^{*} \\
\text { - Extraversion: }-0.20^{* *} \\
\text { Openness: } 0.00 \\
\text { - Agreeableness: } 0.02 \\
\text { - Conscientiousness: } \\
-0.04\end{array}$ & $\begin{array}{l}\text { - Neuroticism: } 0.47^{*} \\
\text { - Extraversion: } 0.03 \\
\text { - Openness: } 0.27^{*} \\
\text { - Agreeableness: } 0.00 \\
\text { - Conscientiousness: } \\
-0.28^{*}\end{array}$ & $\begin{array}{l}\text { - Neuroticism: }-0.33^{*} \\
\text { - Extraversion: } 0.46^{*} \\
\text { - Openness: } 0.12 \\
\text { - Agreeableness: }-0.19 \\
\text { - Conscientiousness: } \\
0.21^{* *}\end{array}$ & $\begin{array}{l}\text { - Neuroticism: } 0.40^{*} \\
\text { - Extraversion: } 0.10 \\
\text { - Openness: } 0.07 \\
\text { - Agreeableness: }-0.34 * \\
\text { - Conscientiousness: } \\
-0.12\end{array}$ & $\begin{array}{l}\text { - Neuroticism: } 0.67^{*} \\
\text { - Extraversion: }-0.06 \\
\text { - Openness: } 0.04 \\
\text { - Agreeableness: } 0.10 \\
\text { - Conscientiousness: }-0.12\end{array}$ \\
\hline $\begin{array}{c}\text { Victor et al. } \\
\text { (2006) }\end{array}$ & NEO-FFI & $\begin{array}{l}\text { Extraversion: }-0.31^{* *} \\
\text { - Neuroticism: } 0.72^{*} \\
\text { - Conscientiousness: } \\
0.01 \\
\text { - Agreeableness: }-0.11 \\
\text { - Openness: }-0.24\end{array}$ & $\begin{array}{l}\text { - Extraversion: }-0.12 \\
\text { - Neuroticism: } 0.33 \\
\text { - Conscientiousness: } \\
-0.09 \\
\text { - Agreeableness: }-0.16 \\
\text { - Openness: }-0.08\end{array}$ & $\begin{array}{l}\text { - Extraversion: } 0.57^{*} \\
\text { - Neuroticism: }-0.46^{*} \\
\text { - Conscientiousness: } \\
0.22 \\
\text { - Agreeableness: }-0.17 \\
\text { - Openness: } 0.17\end{array}$ & $\begin{array}{l}\text { Extraversion: } 0.08 \\
\text { - Neuroticism: } 0.28 \\
\text { - Conscientiousness: } \\
0.06 \\
\text { - Agreeableness: }-0.37^{*} \\
\text { - Openness: }-0.20\end{array}$ & $\begin{array}{l}\text { - Extraversion: }-0.15 \\
\text { - Neuroticism: } 0.49 \\
\text { - Conscientiousness: } 0.13 \\
\text { - Agreeableness: }-0.07 \\
\text { - Openness: }-0.16\end{array}$ \\
\hline $\begin{array}{l}\text { Bloink et al. } \\
\quad(2005)\end{array}$ & NEO-FFI & $\begin{array}{l}\text { - Neuroticism: } 0.56 \\
\text { - Extraversion: }-0.44 \\
\text { - Openness: }-0.09 \\
\text { - Agreeableness: } 0.07 \\
\text { - Conscientiousness: } \\
0.13\end{array}$ & $\begin{array}{l}\text { - Neuroticism: } 0.65 \\
\text { - Extraversion: }-0.18 \\
\text { - Openness: } 0.12 \\
\text { - Agreeableness: }-0.27 \\
\text { - Conscientiousness: } \\
-0.33\end{array}$ & $\begin{array}{ll}\text { - Neuroticism: }-0.37 \\
\text { - Extraversion: } 0.63 \\
\text { - Openness: } 0.14 \\
\text { - } \text { Agreeableness: }-0.06 \\
\text { - Conscientiousness: } \\
0.18\end{array}$ & $\begin{array}{l}\text { - Neuroticism: } 0.41 \\
\text { - Extraversion: }-0.11 \\
\text { - Openness: }-0.02 \\
\text { - Agreeableness: }-0.53 \\
\text { - Conscientiousness: } \\
-0.05\end{array}$ & $\begin{array}{l}\text { - Neuroticism: } 0.76 \\
\text { - Extraversion: }-0.30 \\
\text { - Openness: }-0.03 \\
\text { - Agreeableness: }-0.11 \\
\text { - Conscientiousness: }-0.02\end{array}$ \\
\hline $\begin{array}{l}\text { Akiskal et al. } \\
\quad(2005 \mathrm{c})\end{array}$ & TCI-125 & $\begin{array}{l}\text { - Novelty seeking: } 0.10 \\
\text { - Harm avoidance: } 0.58 \\
\text { - Reward dependence: } \\
-0.21 \\
\text { - Persistence: }-0.17 \\
\text { - Self-directedness: } \\
-0.65 \\
\text { - Cooperativeness: }\end{array}$ & $\begin{array}{l}\text { - Novelty seeking: } 0.35 \\
\text { - Harm avoidance: } 0.49 \\
\text { - Reward dependence: } \\
-0.19 \\
\text { - Persistence: }-0.04 \\
\text { - Self-directedness: } \\
0.42 \\
\text { - Cooperativeness: }\end{array}$ & $\begin{array}{l}\text { - Novelty seeking: } 0.29 \\
\text { - Harm avoidance: } \\
-0.53 \\
\text { - } \text { Reward dependence: } \\
0.14 \\
\text { - Persistence: } 0.31 \\
\text { - Self-directedness: } \\
0.25\end{array}$ & $\begin{array}{l}\text { - Novelty seeking: } 0.26 \\
\text { - Harm avoidance: } 0.32 \\
\text { - Reward dependence: } \\
-0.20 \\
\text { - Persistence: }-0.05 \\
\text { - Self-directedness: } \\
-0.48 \\
\text { - Cooperativeness: }\end{array}$ & $\begin{array}{l}\text { - Novelty seeking: }-0.14 \\
\text { - Harm avoidance: } 0.48 \\
\text { - Reward dependence: } 0.05 \\
\text { - } \text { Persistence: } 0.07 \\
\text { - } \text { Cooperativectedness: }-0.37 \\
\text { - Self-transcendence: } 0.20\end{array}$ \\
\hline
\end{tabular}


Table 3 (continued)

\begin{tabular}{|c|c|c|c|c|c|c|}
\hline \multirow[t]{2}{*}{ Reference } & \multirow{2}{*}{$\begin{array}{l}\text { Scales } \\
\text { used }\end{array}$} & \multicolumn{5}{|c|}{ Correlation with TEMPS temperament score } \\
\hline & & Depressive & Cyclothymic & Hyperthymic & Irritable & Anxious \\
\hline & & $\begin{array}{ll}-0.39 \\
\text { - Self-transcendence: } \\
0.01\end{array}$ & $\begin{array}{ll}-0.32 \\
\text { Self-transcendence: } \\
0.29\end{array}$ & $\begin{array}{l}\text { - Cooperativeness: } 0.07 \\
\text { - Self-transcendence: } \\
0.22\end{array}$ & $\begin{array}{ll}-0.40 \\
\text { - Self-transcendence: } \\
0.09\end{array}$ & \\
\hline
\end{tabular}

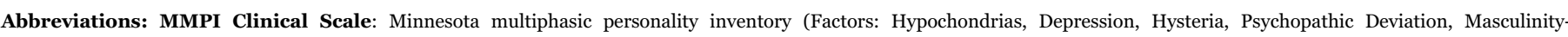

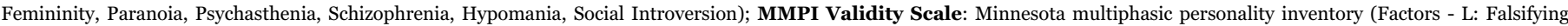

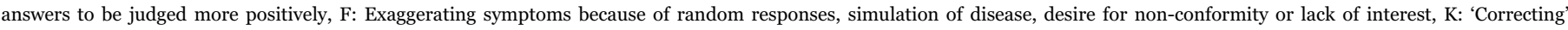

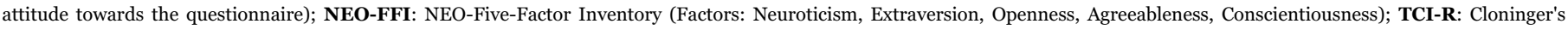

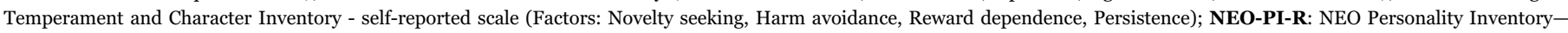

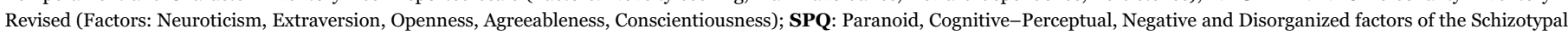

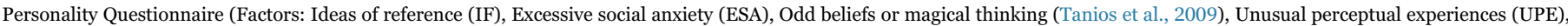

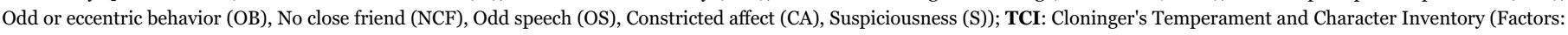
Novelty seeking, Harm avoidance, Reward dependence, Persistence, Self-directedness, Cooperativeness, Self-transcendence).

a The Anxious factor was subdivided into Anxious Cognitive and Anxious Somatic.

* $\mathrm{p}<0.01$.

$\mathrm{p}<0.05$.

\section{(21.5\% of the variance).}

Two studies found a 3-factor structure. These studies reported a low proportion of the variance explained by the factors $(<20 \%)$. Pompili et al. (2008) found a first factor composed by the dysthymic, cyclothymic and anxious temperaments. The two remaining factors were composed by the irritable and hyperthymic temperaments, respectively. Akiyama et al. (2005) did not observe a clear-cut loading of individual items in their 3-factor solution. Factor 1 included 1 depressive, 11 cyclothymic and 12 irritable temperament items, factor 2 included 1 depressive and 10 hyperthymic temperament items, and factor 3 included only two depressive temperament items.

The study from Akiskal et al. (2005b) used factor analysis to develop a shorter version of the TEMPS-A. A four-factor structure for the TEMPS-A was verified with the cyclothymic explaining $14 \%$ of the variance, while the irritable, hyperthymic, and dysthymic explained in combination an additional $14 \%$ of the variance in TEMPS-A scores. A further factor analysis using PCA showed 2 'super factors', with cyclothymic, irritable, and dysthymic temperaments loading on Factor I, and the hyperthymic temperament loading heavily on Factor II. Finally, Akiskal et al. (2005a) found a global factor structure. Before rotation, the data showed one global factor for each subscale, in which nearly all items were well represented. The depressive, cyclothymic and hyperthymic temperaments loaded saliently on this factor. The factor loadings after rotation were not provided.

All studies that validated the TEMPS-A-39 have performed PCA. The original study from Akiskal et al. (2005c) used varimax rotation, while the translations from Yuan et al. (2015) and Preti et al. (2010) used the promax method. All versions found the 5-factor structure proposed for the TEMPS-A-39 instrument.

The study from Maremmani et al. (2011) that validated the TEMPS-A[P] did not evaluate its factor structure.

\subsection{Confirmatory factor analysis (CFA)}

CFA is used to test how well a set of measures fit to a given construct. Equations are built considering a hypothesis defined $a$ priori on how each item of the scale are related to each construct. For each model, goodness-of-fit statistics are calculated to describe how well the model fits the data. Chi-square $\left(\chi^{2}\right)$ is the traditional fit index for evaluating overall model fit, since it quantifies the discrepancy between the sample and fitted models ( $\mathrm{Hu}$ and Bentler, 1999). However, a $\chi^{2}$ likelihood ratio test may be biased by sample size (Tanaka, 1993). The ratio $\chi^{2} /$ degrees of freedom (df) can be used in addition to evaluate model fitting, with ratios larger than 3 indicating poor fit (Byrne, 1989). Other indexes include the comparative fit index (CFI), the goodness-of-fit index (GFI), the adjusted goodness-of-fit index (AGFI), the root mean square error of approximation (RMSEA), the probability test of RMSEA below a threshold (PCLOSE, p-value for RMSEA $\leq 0.05$ ) and the standardized root mean square residual (SRMR). Although no consensus exists in the literature RMSEA $\leq 0.06$, SRMR $\leq 0.09$ and CFI $\geq 0.90$ are considered acceptable (i.e., indicative of good model fit) (Baumgartner and Homburg, 1996; Browne and Cudeck, 1993; Hu and Bentler, 1999). The Akaike information criterion (AIC) is used to compare different models, and the model with the lowest AIC is judged to fit the data better than alternative solutions (Akaike, 1974).

Three studies performed CFA. Ristic-Ignjatovic et al. (2014) extracted two best fitted models for the Serbian version of the TEMPS-A-110. In the five-factor model the anxiety subscale was composed mainly by somatic items $\left(\chi^{2} / \mathrm{df}=2.228\right.$, $p<0.01, \quad$ GFI $=0.892, \quad$ AGFI $=0.876, \quad$ CFI $=0.850, \quad$ RMSEA $=0.046$, PCLOSE=0.952). A six-factor model, where the cognitive (worrying) and the somatic anxious subscales were clearly separated provided a better fit $\left(\chi^{2} / \mathrm{df}=2.002, p<0.01\right.$, GFI $=0.905$, AGFI $=0.898, \mathrm{CFI}=0.887$, RMSEA $=0.032$, PCLOSE $=1.000$ ). The authors found a certain overlap between items pertaining to the worrying dimension and the ones related to the depressive temperament, and the inclusion of somatic anxiety as a distinct factor provided a more accurate model to fit their data.

Fountoulakis et al. (2014) evaluated three models through CFA for the TEMPS-A-110 in a general Greek population. The CFI for the fivefactor model was better than the CFI for the one-factor model ( 0.91 vs. 0.87). Other indexes confirmed that the original five-factor solution was the best fitted model $\left(\chi^{2} / \mathrm{df}=3.6, \mathrm{CFI}=0.944\right.$, RMSEA $=0.060,95 \%$ $\mathrm{CI}=0.057-0.063$, SRMR=0.60, $\mathrm{AIC}=1016.920)$.

Preti et al. (2013) evaluated four models through CFA for the short 39-item TEMPS-A in an Italian sample. The expected five-factor model had the best fit as indicated by all CFA indexes across both genders, age distributions and levels of psychological distress in their sample $\left(\chi^{2}\right)$ $\mathrm{df}=1.7, \mathrm{CFI}=0.927$, RMSEA $=0.035,90 \% \mathrm{CI}=0.032-0.038$ ). The unidimensional model was rejected on the basis of the CFA fit indexes, as were the two- and three-factor models.

\subsection{Internal consistency reliability}

Internal consistency reliability measures how well the scores for individual items on an instrument correlate with each other. Twentythree studies (85.2\%) assessed this property (Table 2). All five studies that validated the TEMPS-A-39 reported acceptable internal consistency, while the studies that validated different TEMPS-A-110 versions (Akiskal et al., 2005a; Akiyama et al., 2005; Kawamura et al., 2010) did not provide Cronbach's alpha values.

Overall, all studies found adequate internal consistency reliabilities 
for versions of the TEMPS-A-110. Median Cronbach's alpha values were: 0.72 (range: $0.62-0.89$ ) for the depressive temperament, 0.81 (range: 0.69-0.89) for the cyclothymic temperament, 0.79 (range: 0.70-0.83) for the hyperthymic temperament, 0.76 (range: 0.70$0.84)$ for the irritable domain, and $0.82(0.67-0.88)$ for the anxiety domain.

For the TEMPS-A-39, median Cronbach's alpha coefficients were $0.73(0.72-0.81)$ for the depressive, $0.83(0.79-0.91)$ for the cyclothymic, $0.72(0.7-0.76)$ for the hyperthymic, and $0.71(0.65-0.77)$ for the irritable temperaments. The internal consistency of the anxious temperament was not acceptable in general, with a median value of $0.67(0.67-0.71)$.

\subsection{Test-retest reliability}

Test-retest reliability measures the temporal stability of an underlying construct. Only 9 studies (1407 subjects; range: 30-426) assessed this property (Akiskal et al., 2005b; Erfurth et al., 2005; Hinic et al., 2013; Kawamura et al., 2010; Lin et al., 2013; Matsumoto et al., 2005; Ristic-Ignjatovic et al., 2014; Vahip et al., 2005; Yuan et al., 2015). The interval between the applications ranged from 2 weeks to 6 years. All but one study evaluated versions of the TEMPS-A-110. The study by Erfurth et al. (2005) evaluated the brief TEMPS-M-35 version, and found a test-retest correlation of 0.72 for the depressive, 0.69 for cyclothymic, 0.49 for hyperthymic, and 0.70 for both irritable and anxious temperaments. The remaining studies that assessed the original TEMPS-A-110 version found median correlations of 0.64 (0.59-0.69) for the depressive, $0.68(0.68-0.68)$ for the cyclothymic, $0.82(0.82-0.82)$ for the hyperthymic, $0.66(0.66-0.66)$ for the irritable and $0.8(0.74-0.86)$ for the anxious temperaments.

Only the study by Yuan et al. (2015) assessed the test-retest reliability of the TEMPS-A-39. They found test-retest correlations of 0.52 for the depressive, 0.85 for the cyclothymic, 0.59 for the hyperthymic, 0.74 for the irritable, and 0.71 for the anxious temperaments.

\subsection{Concurrent validity}

Seven studies compared the TEMPS measures with other validated instruments related to personality constructs (Akiskal et al., 2005c; Bloink et al., 2005; Maremmani et al., 2011; Preti et al., 2015; RisticIgnjatovic et al., 2014; Rozsa et al., 2008; Victor et al., 2006) (Table 3).

Of the four studies that evaluated versions of TEMPS-A-110, RisticIgnjatovic et al. (2014) and Rozsa et al. (2008) compared affective temperaments with versions of Cloninger's Temperament and Character Inventory (TCI) dimensions (Cloninger et al., 1994; Dzamonja-Ignjatovic et al., 2010). Consistent findings show that the harm avoidance dimension is negatively associated with hyperthymic temperament scores, and positively associated with both depressive and anxious temperament scores. The studies of Bloink et al. (2005), Rozsa et al. (2008) and Victor et al. (2006) used versions of the NEO Personality Inventory (NEO-PI-R and NEO-FFI) (Costa Jr and McCrae, 1992). Overall, neuroticism was positively associated predominantly with the TEMPS depressive and anxious temperaments, and negatively correlated with the hyperthymic temperament. Agreeableness was negatively associated with the irritable temperament. Finally, extraversion was positively associated with hyperthymic temperament and negatively correlated with the depressive temperament.

Akiskal et al. (2005c) also used the TCI to assess concurrent validity in the short TEMPS-A-39. Findings were similar to the other studies that compared TCI with the 110-version of TEMPS-A. Preti et al. (2015) specifically assessed concurrent validity using the Schizotypal Personality Questionnaire (Raine, 1991). The hyperthymic temperament had lower correlations with ideas of reference, excessive social anxiety, unusual perceptual experiences, no close friend and odd speech. Suspiciousness was higher in the other temperaments, especially in the depressive and anxious affective temperaments. Unusual perceptual experiences and ideas of reference were also higher in the cyclothymic temperament.

The study of Maremmani et al. (2011) that assessed the TEMPS$\mathrm{A}[\mathrm{P}]$ used the Minnesota Multiphasic Personality Inventory (Hathaway et al., 1989). Although several correlations were not reported, the hyperthymic temperament was significantly associated with hypomania.

\section{Discussion}

This systematic review indicated that several versions of the TEMPS (e.g., TEMPS-I, TEMPS-A-110, and the 39-item version of the TEMPSA) are available. In addition, the TEMPS instrument has been validated for use in adult samples across 14 different languages and 15 countries. Notwithstanding the developers of this set of instruments aimed to identify five distinct affective temperaments (i.e., hyperthymic, cyclothymic, irritable, depressive, and anxious temperaments) (Akiskal and Akiskal, 2005; Akiskal et al., 2005b, 2005c), some validation efforts failed to replicate this factor structure (vide infra). Furthermore, different adapted versions of the TEMPS instrument have been developed for use in specific cultures (for example, the 45-item TEMPS-Rio de Janeiro and the Münster 35-item version of the TEMPS-A) (Erfurth et al., 2005; Woodruff et al., 2011). These findings point to possible culture-bound influences in the assessment of affective temperaments. In this regard, Vazquez et al. (2012) performed a combined analysis of general population samples from different countries, and found that the prevalence of affective temperaments may display both universal and culture-specific findings. This aspect arguably imposes an obstacle to the comparability of research findings across cultures.

\subsection{Validity of different TEMPS versions}

Several validation studies have performed exploratory PCA of different versions of the TEMPS. For the TEMPS-A-110 not all studies found the expected five-factor structure. A six-factor solution in which the anxiety factor was split into a 'cognitive' and a 'somatic' component was observed for the Serbian version of the TEMPS-A-110 (RisticIgnjatovic et al., 2014). A three-factor solution was observed for Italian and French versions of the TEMPS-A-110 (Akiskal et al., 2005a; Pompili et al., 2008). Alternative two-factors solutions were reported for Chinese, Hungarian, and Polish version of the TEMPS-A-110 (Borkowska et al., 2010; Lin et al., 2013; Rozsa et al., 2008). Notwithstanding cultural peculiarities may have contributed to these differences, one should consider that these studies enrolled heterogeneous samples from different settings. In addition, PCA is exploratory in nature, and relatively fewer studies have performed CFA. RisticIgnjatovic et al. (2014) found a six-factor structure for the Serbian version of the TEMPS-A-110 (i.e., with 'somatic' and a 'cognitive' factor for the anxiety temperament), while a general population study conducted in a Greek sample confirmed its original five-factor solution (Fountoulakis et al., 2014). Interestingly, all studies that conducted exploratory PCA of the 39-item version of the TEMPS-A found the expected five-factor solution (Akiskal et al., 2005c; Preti et al., 2010; Yuan et al., 2015). Furthermore, this five-factor solution was further supported by CFA in an Italian student sample (Preti et al., 2013).

For concurrent validity different dimensions of the TEMPS were compared to the Cloninger's psychobiological model of temperament (Cloninger et al., 1994) and the five-factor model (FFM) of personality (Costa Jr and McCrae, 1992) in at least two studies. The hyperthymic temperament was consistently associated with lower scores in harm avoidance, while this dimension was positively associated with the anxious and depressive dimensions of the TEMPS (Akiskal et al., 2005b; Ristic-Ignjatovic et al., 2014; Rozsa et al., 2006). The neuroticismdimension of the FFM was negatively associated with the 
hyperthymic dimension, and positively associated with the depressive and anxious dimensions of the TEMPS (Bloink et al., 2005; RisticIgnjatovic et al., 2014; Rozsa et al., 2008), while expectedly the agreeableness dimension of the FFM was consistently associated with the TEMPS irritable dimension. Finally, the extraversion dimension of the FFM was positively associated with the hyperthymic dimension, and negatively associated with scores of the depressive temperament.

These findings seem consistent with the idea that the hyperthymic temperament may be a more resilient temperament (Carvalho et al., 2013; Karam et al., 2010; Kesebir et al., 2013; Vazquez et al., 2010). This interpretation is further supported by the fact that the hyperthymic temperament was negatively associated with neuroticism, which is a personality dimension consistently associated with psychopathology (Jeronimus et al., 2016). In addition, a recent meta-analysis indicates that hyperthymic temperament scores were higher in healthy controls compared to patients with mood disorder on a continuum (Solmi et al., 2016).

\subsection{Reliability of different TEMPS versions}

Overall, this systematic review indicates that the different versions and dimensions of the TEMPS have adequate internal consistency reliabilities. However, relatively few studies have evaluated the testretest reliability of different versions of the TEMPS, and results thus far have not been conclusive. The TEMPS-A-110 version has adequate test-retest reliability, while the only study that evaluated the test-retest reliability of the 39-item TEMPS-A version found low correlation coefficients for the hyperthymic and depressive dimensions (Yuan et al., 2015). The study by Kawamura et al. (2010) found robust sixyear test-retest correlation coefficients for all TEMPS-A-110 dimensions in a convenience sample of Japanese white-collar workers.

\subsection{Limitations}

Some limitations of this systematic review warrant discussion. First, we excluded validation studies that enrolled non-adult samples. This decision was made because some evidence indicates that temperaments may not be stable construct during childhood (Nigg and Goldsmith, 1998; Saudino, 2005). Second, we included only peerreviewed reports. Whilst this could also be considered a strength since this ensures articles have been peer reviewed, it means that we may have missed studies from the 'gray literature'. On the other hand, the inclusion of peer-reviewed studies might indicate that the most methodologically rigorous studies were herein included. Third, the methodological quality of included studies varied in this literature with the inclusion of heterogeneous samples from diverse settings. In addition, our methodological assessment tool has not been previously validated. Finally, a meta-analysis was not performed due to the heterogeneity of samples and settings, and the use of different versions of the TEMPS.

\subsection{Clinical and research implications}

This systematic review indicates that affective temperaments as assessed by the TEMPS-A-110 version do not universally fit the proposed five-factor model. Thus, comparisons of findings across studies performed across different cultures may be challenging. A five-factor solution for the 39-item version of the TEMPS-A has been consistently reported although this version of the instruments has been less extensively investigated. The concept of predominant affective temperament has been proposed. Typically, studies have considered a threshold of +2 SD or a score above the 95th percentile in each TEMPS dimension to operationalize a dominant temperament (Fountoulakis et al., 2014; Karam et al., 2005). However, the frequency of different affective temperaments may vary with these two different approaches (Fountoulakis et al., 2014). In addition, in some samples most participants may not exhibit a Z-score above $2 \mathrm{SD}$ in the TEMPS (e.g., (Karam et al., 2005)). Furthermore, it is worthy to note that there are persuasive arguments in the literature against arbitrarily dichotomizing measures of continuous constructs (MacCallum et al., 2002). A recent systematic review and meta-analysis indicated that scores of some TEMPS-derived affective temperaments may occur on a continuum or gradient in individuals with major depressive disorder (MDD), bipolar disorder (BD), and healthy controls (Solmi et al., 2016). A dimensional approach for the TEMPS was further supported by genome-wide association study that found a significant robust genome-wide association for the irritable temperament in a large sample with BD (Greenwood et al., 2012). The authors of this study hypothesized that a dimensional approach for the TEMPS may provide more useful information for the genetic subtyping of affective disorders.

\section{Conclusion}

In conclusion, this systematic review indicates that several versions of the TEMPS instrument are available. However, the factor structure of the TEMPS-A-110 version seems to vary across different studies. A shorter version 39-item version of the TEMPS-A holds promise as a validated measure for the assessment of affective temperaments. Therefore, this shorter version of the TEMPS may offer significant advantages (e.g. enhancing the compliance of research participants), while also capturing the underlying construct evaluated by longer versions of the TEMPS. Nevertheless, this version has been less extensively studied than the TEMPS-A-110 version in different cultures. Although the assessment of affective temperaments may continue to open important insights for the psychopathological comprehension of affective disorders, while also contributing to the subtyping of these heterogeneous phenotypes on a dimensional basis, further work is required to validate and standardize shorter versions of the TEMPS across cultures.

\section{Appendix A. Supporting information}

Supplementary data associated with this article can be found in the online version at doi:10.1016/j.jad.2017.01.023.

\section{References}

Akaike, H., 1974. A new look at the statistical model identification. IEEE Trans. Autom. Control 19, 716-723.

Akiskal, H.S., Mallya, G., 1987. Criteria for the "soft" bipolar spectrum: treatment implications. Psychopharmacol. Bull. 23, 68-73.

Akiskal, H.S., Pinto, O., 1999. The evolving bipolar spectrum. prototypes I, II, III, and IV Psychiatr. Clin. North Am. 22, 517-534, (vii).

Akiskal, H.S., Akiskal, K.K., 2005. TEMPS: Temperament Evaluation of Memphis, Pisa, Paris and San Diego. J. Affect. Disord. 85, 1-2.

Akiskal, H.S., Akiskal, K.K., 2007. In search of Aristotle: temperament, human nature, melancholia, creativity and eminence. J. Affect. Disord. 100, 1-6.

Akiskal, H.S., Placidi, G.F., Maremmani, I., Signoretta, S., Liguori, A., Gervasi, R. Mallya, G., Puzantian, V.R., 1998. TEMPS-I: delineating the most discriminant traits of the cyclothymic, depressive, hyperthymic and irritable temperaments in a nonpatient population. J. Affect. Disord. 51, 7-19.

Akiskal, H.S., Akiskal, K., Allilaire, J.F., Azorin, J.M., Bourgeois, M.L., Sechter, D., Fraud, J.P., Chatenet-Duchene, L., Lancrenon, S., Perugi, G., Hantouche, E.G., 2005a. Validating affective temperaments in their subaffective and socially positive attributes: psychometric, clinical and familial data from a French national study. J. Affect. Disord. 85, 29-36.

Akiskal, H.S., Akiskal, K.K., Haykal, R.F., Manning, J.S., Connor, P.D., 2005b. TEMPS-A progress towards validation of a self-rated clinical version of the Temperament Evaluation of the Memphis, Pisa, Paris, and San Diego Autoquestionnaire. J. Affect. Disord. 85, 3-16.

Akiskal, H.S., Mendlowicz, M.V., Jean-Louis, G., Rapaport, M.H., Kelsoe, J.R., Gillin, J.C., Smith, T.L., 2005c. TEMPS-A: validation of a short version of a self-rated instrument designed to measure variations in temperament. J. Affect. Disord. 85, $45-52$.

Akiskal, K.K., Savino, M., Akiskal, H.S., 2005d. Temperament profiles in physicians, lawyers, managers, industrialists, architects, journalists, and artists: a study in psychiatric outpatients. J. Affect. Disord. 85, 201-206.

Akiyama, T., Tsuda, H., Matsumoto, S., Miyake, Y., Kawamura, Y., Noda, T., Akiskal, 
K.K., Akiskal, H.S., 2005. The proposed factor structure of temperament and personality in Japan: combining traits from TEMPS-A and MPT. J. Affect. Disord. 85, 93-100.

Angst, J., 2007. The bipolar spectrum. Br. J. Psychiatry.: J. Ment. Sci. 190, 189-191.

Bakkalbasi, N., Bauer, K., Glover, J., Wang, L., 2006. Three options for citation tracking: Google Scholar, Scopus and Web of Science. Biomed. Digit. Libr. 3, 7.

Baldessarini, R.J., Vazquez, G.H., Tondo, L., 2016. Affective temperaments and suicidal ideation and behavior in mood and anxiety disorder patients. J. Affect. Disord. 198, $78-82$.

Baumgartner, H., Homburg, C., 1996. Applications of structural equation modeling in marketing and consumer research: a review. Int. J. Res. Mark. 13, 139-161.

Bloink, R., Brieger, P., Akiskal, H.S., Marneros, A., 2005. Factorial structure and internal consistency of the German TEMPS-A scale: validation against the NEO-FFI questionnaire. J. Affect. Disord. 85, 77-83.

Borkowska, A., Rybakowski, J.K., Drozdz, W., Bielinski, M., Kosmowska, M., RajewskaRager, A., Bucinski, A., Akiskal, K.K., Akiskal, H.S., 2010. Polish validation of the TEMPS-A: the profile of affective temperaments in a college student population. J. Affect. Disord. 123, 36-41.

Browne, M.W., Cudeck, R., 1993. Alternative ways of assessing model fit. In: Bollen, K.A., Long, J.S. (Eds.), Testing Structural Equation Models.. Sage, Newbury Park, CA, $136-161$.

Byrne, B.M., 1989. A Primer of LISREL: basic Applications and Programming for Confirmatory Factor Analytic Model. Springer-Verlag, New York.

Carvalho, A.F., Hyphantis, T.N., Taunay, T.C., Macedo, D.S., Floros, G.D., Ottoni, G.L., Fountoulakis, K.N., Lara, D.R., 2013. The relationship between affective temperaments, defensive styles and depressive symptoms in a large sample. J. Affect. Disord. 146, 58-65.

Cloninger, C.R., Przybeck, T.R., Svrakic, D.M., 1994. The Temperament and Character Inventory (TCI): a guide to its development and useCenter for Psychobiology of Personality. Washington University St. Louis, MO.

Costa, P., Jr, McCrae, R.R., 1992. Revised NEO personality inventory (NEO-PI-R) and NEO five-factor (NEO-FFI) inventory professional manual. PAR, Odessa, FL.

Dolenc, B., Sprah, L., 2011. Psychometric characteristics of the long version of Slovenian temperament autoquestionnaire TEMPS-A (Temperament Evaluation of Memphis, Pisa, Paris and San Diego-Autoquestionnaire). Psihol. Obz. / Horiz. Psychol. 20, $5-24$.

Dolenc, B., Sprah, L., Dernovsek, M.Z., Akiskal, K., Akiskal, H.S., 2013. Psychometric properties of the Slovenian version of temperament evaluation of Memphis, Pisa, Paris, and San Diego - Autoquestionnaire (TEMPS-A): Temperament profiles in Slovenian university students. J. Affect. Disord. 144, 253-262.

Dzamonja-Ignjatovic, T., Svrakic, D.M., Svrakic, N., Jovanovic, M.D., Cloninger, R.C., 2010. Cross-cultural validation of the revised Temperament and Character Inventory: Serbian data. Compr. Psychiatry 51, 649-655.

Erfurth, A., Gerlach, A.L., Hellweg, I., Boenigk, I., Michael, N., Akiskal, H.S., 2005. Studies on a German (Munster) version of the temperament auto-questionnaire TEMPS-A: construction and validation of the briefTEMPS-M. J. Affect. Disord. 85, $53-69$.

Figueira, M.L., Caeiro, L., Ferro, A., Severino, L., Duarte, P.M., Abreu, M., Akiskal, H.S., Akiskal, K.K., 2008. Validation of the Temperament Evaluation of Memphis, Pisa, Paris and San Diego (TEMPS-A): Portuguese-Lisbon version. J. Affect. Disord. 111, 193-203.

Fountoulakis, K.N., Siamouli, M., Magiria, M., Pantoula, E., Moutou, K., Kemeridou, M., Mavridou, E., Panagiotidis, P., Loli, E., Batsiari, E., Preti, A., Tondo, L., Gonda, X., Rihmer, Z., Akiskal, K., Akiskal, H., 2014. Standardization of the TEMPS-A in the Greek general population. J. Affect. Disord. 158, 19-29.

Fountoulakis, K.N., Gonda, X., Koufaki, I., Hyphantis, T., Cloninger, C.R., 2016. The role of temperament in the Etiopathogenesis of bipolar spectrum illness. Harv. Rev. Psychiatry 24, 36-52.

Ghaemi, S.N., Dalley, S., 2014. The bipolar spectrum: conceptions and misconceptions. Aust. N.Z. J. Psychiatry 48, 314-324.

Greenwood, T.A., Akiskal, H.S., Akiskal, K.K., Kelsoe, J.R., 2012. Genome-wide association study of temperament in bipolar disorder reveals significant associations with three novel Loci. Biol. Psychiatry 72, 303-310.

Hathaway, S.R., McKinley, J.C., Committee, M.R., 1989. MMPI-2: minnesota Multiphasic Personality Inventory-2: manual for administration and scoring. University of Minnesota Press.

Hinic, D., Akiskal, S.H., Akiskal, K.K., Jovic, J., Ignjatovic Ristic, D., 2013. Validation of the Temps-A in university student population in Serbia. J. Affect. Disord. 149, $146-151$.

Hu, Lt, Bentler, P.M., 1999. Cutoff criteria for fit indexes in covariance structure analysis: conventional criteria versus new alternatives. Struct. Equ. Model.: a Multidiscip. J. 6, $1-55$.

Hyphantis, T.N., Taunay, T.C., Macedo, D.S., Soeiro-de-Souza, M.G., Bisol, L.W., Fountoulakis, K.N., Lara, D.R., Carvalho, A.F., 2013. Affective temperaments and ego defense mechanisms associated with somatic symptom severity in a large sample. J. Affect. Disord. 150, 481-489.

Jeronimus, B.F., Kotov, R., Riese, H., Ormel, J., 2016. Neuroticism's prospective association with mental disorders halves after adjustment for baseline symptoms and psychiatric history, but the adjusted association hardly decays with time: a metaanalysis on 59 longitudinal/prospective studies with 443313 participants. Psychol. Med., 1-24.

Karam, E.G., Mneimneh, Z., Salamoun, M., Akiskal, K.K., Akiskal, H.S., 2005 Psychometric properties of the Lebanese-Arabic TEMPS-A: a national epidemiologic study. J. Affect. Disord. 87, 169-183.

Karam, E.G., Salamoun, M.M., Yeretzian, J.S., Mneimneh, Z.N., Karam, A.N., Fayyad, J., Hantouche, E., Akiskal, K., Akiskal, H.S., 2010. The role of anxious and hyperthymic temperaments in mental disorders: a national epidemiologic study. World Psychiatry.: Off. J. World Psychiatr. Assoc. (WPA) 9, 103-110.

Kawamura, Y., Akiyama, T., Shimada, T., Minato, T., Umekage, T., Noda, Y., Ukawa, K. Hashidume, C., Sakai, Y., Otowa, T., Sasaki, T., Akiskal, H.S., 2010. Six-year stability of affective temperaments as measured by TEMPS-A. Psychopathology 43, 240-247.

Kesebir, S., Gundogar, D., Kucuksubasi, Y., Tatlidil Yaylaci, E., 2013. The relation between affective temperament and resilience in depression: a controlled study. J. Affect. Disord. 148, 352-356.

Kraepelin, E., 1921. Manic depressive insanity and paranoia. J. Nerv. Ment. Dis. 53, 350.

Krebs, M.O., Kazes, M., Olie, J.P., Loo, H., Akiskal, K., Akiskal, H., 2006. The French version of the validated short TEMPS-A: the temperament evaluation of Memphis, Pisa, Paris and San Diego. J. Affect. Disord. 96, 271-273.

Kretschmer, E., 1936. Psychique und Character. Kegan, Paul, Trench, Trubner and Co, Ltd., London UK.

Lin, K., Xu, G., Miao, G., Ning, Y., Ouyang, H., Chen, X., Hoang, N., Akiskal, K.K., Akiskal, H.S., 2013. Psychometric properties of the Chinese (Mandarin) TEMPS-A: a population study of 985 non-clinical subjects in China. J. Affect. Disord. 147, 29-33.

MacCallum, R.C., Zhang, S., Preacher, K.J., Rucker, D.D., 2002. On the practice of dichotomization of quantitative variables. Psychol. Methods 7, 19-40.

Maremmani, I., Dell'Osso, L., Rovai, L., Arduino, G., Montagnari, A., Abbenante, D., Popovic, D., Maremmani, A.G., Perugi, G., Akiskal, K., Akiskal, H.S., 2011. Discriminant and convergent validity of TEMPS-A[P] correlation with MMPI and the emotional-affective state following a stressful situation. J. Affect. Disord. 129, 27-33.

Matsumoto, S., Akiyama, T., Tsuda, H., Miyake, Y., Kawamura, Y., Noda, T., Akiskal, K.K., Akiskal, H.S., 2005. Reliability and validity of TEMPS-A in a Japanese nonclinical population: application to unipolar and bipolar depressives. J. Affect. Disord. 85, 85-92.

Moher, D., Liberati, A., Tetzlaff, J., Altman, D.G., 2009. Preferred reporting items for systematic reviews and meta-analyses: the PRISMA statement. Ann. Intern. Med. 151 (264-269), w264.

Naderer, A., Keller, F., Plener, P., Unseld, M., Lesch, O.M., Walter, H., Erfurth, A., Kapusta, N.D., 2015. The brief TEMPS-M temperament questionnaire: a psychometric evaluation in an Austrian sample. J. Affect. Disord. 188, 43-46.

Nigg, J.T., Goldsmith, H.H., 1998. Developmental psychopathology, personality, and temperament: reflections on recent behavioral genetics research. Hum. Biol. 70, $387-412$.

Perugi, G., Toni, C., Maremmani, I., Tusini, G., Ramacciotti, S., Madia, A., Fornaro, M., Akiskal, H.S., 2012. The influence of affective temperaments and psychopathological traits on the definition of bipolar disorder subtypes: a study on bipolar I Italian national sample. J. Affect. Disord. 136, e41-e49.

Placidi, G.F., Signoretta, S., Liguori, A., Gervasi, R., Maremmani, I., Akiskal, H.S., 1998 The semi-structured affective temperament interview (TEMPS-I). reliability and psychometric properties in 1010 14-26-year old students. J. Affect. Disord. 47, 1-10.

Pompili, M., Girardi, P., Tatarelli, R., Iliceto, P., De Pisa, E., Tondo, L., Akiskal, K.K., Akiskal, H.S., 2008. TEMPS-A (Rome): psychometric validation of affective temperaments in clinically well subjects in mid- and south Italy. J. Affect. Disord. $107,63-75$.

Preti, A., Vellante, M., Zucca, G., Tondo, L., Akiskal, K., Akiskal, H., 2010. The Italian version of the validated short TEMPS-A: the temperament evaluation of Memphis, Pisa, Paris and San Diego. J. Affect. Disord. 120, 207-212.

Preti, A., Vellante, M., Gabbrielli, M., Lai, V., Muratore, T., Pintus, E., Pintus, M., Sanna, S., Scanu, R., Tronci, D., Corrias, I., Petretto, D.R., Carta, M.G., 2013. Confirmatory factor analysis and measurement invariance by gender, age and levels of psychological distress of the short TEMPS-A. J. Affect. Disord. 151, 995-1002.

Preti, A., Corrias, I., Gabbrielli, M., Lai, V., Muratore, T., Pintus, E., Pintus, M., Sanna, S. Scanu, R., Tronci, D., Vellante, M., Siddi, S., Petretto, D.R., Carta, M.G., 2015. The independence of schizotypy from affective temperaments-a combined confirmatory factor analysis of SPQ and the short TEMPS-A. Psychiatry Res. 225, 145-156.

Raine, A., 1991. The SPQ: a scale for the assessment of schizotypal personality based on DSM-III-R criteria. Schizophr. Bull. 17, 555.

Ristic-Ignjatovic, D., Hinic, D., Bessonov, D., Akiskal, H.S., Akiskal, K.K., Ristic, B., 2014. Towards validation of the short TEMPS-A in non-clinical adult population in Serbia. J. Affect. Disord. 164, 43-49.

Rozsa, S., Rihmer, A., Ko, N., Gonda, X., Szili, I., Szadoczky, E., Pestality, P., Rihmer, Z., 2006. [Affective temperaments: psychometric properties of the Hungarian TEMPSA]. Psychiatr. Hung.: A Magy. Pszichiatr. Tars. Tud. F. 21, 147-160.

Rozsa, S., Rihmer, Z., Gonda, X., Szili, I., Rihmer, A., Ko, N., Nemeth, A., Pestality, P., Bagdy, G., Alhassoon, O., Akiskal, K.K., Akiskal, H.S., 2008. A study of affective temperaments in Hungary: internal consistency and concurrent validity of the TEMPS-A against the TCI and NEO-PI-R. J. Affect. Disord. 106, 45-53.

Sanchez-Moreno, J., Barrantes-Vidal, N., Vieta, E., Martinez-Aran, A., Saiz-Ruiz, J. Montes, J.M., Akiskal, K., Akiskal, H.S., 2005. Process of adaptation to Spanish of the temperament Evaluation of Memphis, Pisa, Paris and San Diego scale. self applied version (TEMPS-A). Actas Esp. De. Psiquiatr. 33, 325-330.

Saudino, K.J., 2005. Behavioral genetics and child temperament. J. Dev. Behav. Pediatr. JDBP 26, 214-223.

Schmidt, A., Rodrigues, R.S., Pipa, C.C., Brandalise, L.N., Lorenzi, T.M., Lara, D.R., 2010. Emotional and affective temperament in 23 professional areas. J. Affect. Disord. 126, 49-54.

Solmi, M., Zaninotto, L., Toffanin, T., Veronese, N., Lin, K., Stubbs, B., Fornaro, M., Correll, C.U., 2016. A comparative meta-analysis of TEMPS scores across mood disorder patients, their first-degree relatives, healthy controls, and other psychiatric disorders. J. Affect. Disord. 196, 32-46.

Srivastava, S., Childers, M.E., Baek, J.H., Strong, C.M., Hill, S.J., Warsett, K.S., Wang, P.W., Akiskal, H.S., Akiskal, K.K., Ketter, T.A., 2010. Toward interaction of affective and cognitive contributors to creativity in bipolar disorders: a controlled study. J. 
Affect. Disord. 125, 27-34.

Tanaka, J.S., 1993. Multifaceted conceptions of fit in structural equation models. In Bollen, K.A., Long, J.S. (Eds.), Testing Structural Equation Models. Sage, Newbury Park, CA, 10-39.

Tanios, C.Y., Abou-Saleh, M.T., Karam, A.N., Salamoun, M.M., Mneimneh, Z.N., Karam, E.G., 2009. The epidemiology of anxiety disorders in the Arab world: a review. J. Anxiety Disord. 23, 409-419.

Vahip, S., Kesebir, S., Alkan, M., Yazici, O., Akiskal, K.K., Akiskal, H.S., 2005. Affective temperaments in clinically-well subjects in Turkey: initial psychometric data on the TEMPS-A. J. Affect. Disord. 85, 113-125.

Vazquez, G.H., Nasetta, S., Mercado, B., Romero, E., Tifner, S., Ramon Mdel, L., Garelli, V., Bonifacio, A., Akiskal, K.K., Akiskal, H.S., 2007. Validation of the TEMPS-A Buenos Aires: Spanish psychometric validation of affective temperaments in a population study of Argentina. J. Affect. Disord. 100, 23-29.

Vazquez, G.H., Gonda, X., Zaratiegui, R., Lorenzo, L.S., Akiskal, K., Akiskal, H.S., 2010 Hyperthymic temperament may protect against suicidal ideation. J. Affect. Disord.
$127,38-42$.

Vazquez, G.H., Tondo, L., Mazzarini, L., Gonda, X., 2012. Affective temperaments in general population: a review and combined analysis from national studies. J. Affect. Disord. 139, 18-22.

Victor, D., Sakado, K., Mundt, C., Kronmuller, K.-T., 2006. Psychometric Evaluation of the German version of the temperament Questionnaire TEMPS-A. PPmP: Psychother. Psychosom. Med. Psychol. 56, 70-77.

Woodruff, E., Genaro, L.T., Landeira-Fernandez, J., Cheniaux, E., Laks, J., Jean-Louis, G., Nardi, A.E., Versiani, M.C., Akiskal, H.S., Mendlowicz, M.V., 2011. Validation of the Brazilian brief version of the temperament auto-questionnaire TEMPS-A: the brief TEMPS-Rio de Janeiro. J. Affect. Disord. 134, 65-76.

Yuan, C., Huang, J., Gao, K., Wu, Z., Chen, J., Wang, Y., Hong, W., Yi, Z., Hu, Y., Cao, L., Li, Z., Akiskal, K.K., Akiskal, H.S., Wang, B., Fang, Y., 2015. Validation of the Chinese Version of the Short TEMPS-A and its application in patients with mood disorders. J. Affect. Disord. 170, 178-184. 